Pacific Journal of Mathematics

SYSTEMS OF NONLINEAR WAVE EQUATIONS WITH 


\section{SYSTEMS OF NONLINEAR WAVE EQUATIONS WITH NONLINEAR VISCOSITY}

\section{AVNER FRIEDMAN AND JINDRICH NECAS}

An equation of the form

$$
\ddot{u}-\sum_{i=1}^{n} \frac{\partial}{\partial x_{l}} \frac{\partial W(p)}{\partial p_{l}}-\sum_{i=1}^{n} \frac{\partial}{\partial x_{l}} \frac{\partial V(q)}{\partial q_{l}}=f
$$

where $p=\nabla u, q=\nabla \dot{u}, \dot{u}=\partial u / \partial t, \ddot{u}=\partial^{2} u / \partial t^{2}$ represents, for suitable functions $W(p), V(q)$, a nonlinear hyperbolic equation with nonlinear viscosity and it appears in models of nonlinear elasticity. In this paper existence and regularity of solutions for the Cauchy problem will be established. In particular, if $n=2$, or if $n \geq 3$ and the eigenvalues of $\left(\partial^{2} V / \partial q, \partial q_{j}\right)$ belong to a "small" interval, then the solution is classical. These results will actually be established for a system of equations of the above type.

Introduction. Consider a system of $N$ nonlinear equations

$$
\ddot{u}_{k}-\sum_{i=1}^{n} \frac{\partial}{\partial x_{i}} \frac{\partial W(p)}{\partial p_{k i}}-\sum_{i=1}^{n} \frac{\partial}{\partial x_{i}} \frac{\partial V(q)}{\partial q_{k i}}=f_{k} \quad(1 \leq k \leq N)
$$

in a cylinder $\Omega \times(0, \infty)$, with initial data

$$
u_{k}(x, 0)=u_{k 0}(x), \quad \dot{u}_{k}(x, 0)=u_{k 1}(x)
$$

and boundary conditions

$$
u=0 \text { if } x \in \partial \Omega, t>0 ;
$$

here $\boldsymbol{\Omega}$ is a bounded domain in $\mathbf{R}^{n}$,

$$
\begin{aligned}
p & =\left(p_{l i}\right), \quad q=\left(q_{l i}\right) \quad \text { and } \\
p_{l i} & =\frac{\partial u_{l}}{\partial x_{i}}, \quad q_{l i}=\frac{\partial \dot{u}_{l}}{\partial x_{i}}, \quad \dot{w}=\frac{\partial w}{\partial t} .
\end{aligned}
$$

The special case

$$
N=1, \quad \sum_{i=1}^{n} \frac{\partial}{\partial x_{i}} \frac{\partial V(q)}{\partial q_{k i}}=\Delta \dot{u} \quad(k=1)
$$

has been studied by several authors. For $n=1$, existence and uniqueness of a classical solution was established in [1], [2], [6], [7]. For 
general $n$ Engler [3] has recently established the existence of a strong solution when $W(p)$ is a general nonlinear isotropic function, that is, $\nabla W(p)=g\left(|p|^{2}\right) p$, and $g(s)$ satisfies:

$$
-\frac{1}{2}<k_{0} \leq \frac{s g^{\prime}(s)}{g(s)} \leq k_{1}<\infty ;
$$

in case $g(s)$ is globally Lipschitz, the solution is classical. His results depend in a crucial way on the assumptions in (0.4) (especially the second one). For earlier work on weak solutions see also the references given in [3].

For $n=2$ Petcher [10] established the smoothness of the weak solutions, in case (0.4) and $\partial W(p) / \partial p_{i}=\sigma_{i}\left(p_{i}\right), \sigma_{i}^{\prime}(s) \geq 0, \sigma_{i}^{\prime}(s) \geq$ $c_{1}|s|^{2}$ if $|s| \geq 1, \sigma_{i}^{\prime \prime}(s) \leq c_{2}|s|$ if $|s| \geq 1$ and $c_{1}, c_{2}$ are positive constants.

Systems of the form (0.1)-(0.3) may be considered to represent models of wave propagation in elastic material with nonlinear Hook's Law (corresponding to the internal energy function $W$ ) and nonlinear viscosity (corresponding to $V$ ). In case (0.4), when $n=1$ the equation models simple shear motion of a beam (see [6]), and when $n=2$ it models antiplane shear motion of a column with cross section $\mathbf{\Omega}$ (see [4], [8]); $u$ is the displacement from the rest position. Nonlinear viscosity terms appear in various models of elasticity; see [11].

In this paper we consider $(0.1)-(0.3)$ with both $W(p)$ and $V(q)$ nonlinear functions. In $\S \S 1-5$ we assume that

$$
\begin{aligned}
& \left(\frac{\partial^{2} W(p)}{\partial p_{k} \partial p_{l_{l}}}\right) \text { and }\left(\frac{\partial^{2}(V(q)}{\partial q_{k l} \partial q_{l_{j}}}\right) \text { are uniformly } \\
& \text { positive definite and bounded matrices. }
\end{aligned}
$$

It is well known that under these conditions there exists a unique global weak solution; our interest is to derive regularity of the solution. In fact we prove (in $\S 5$ ) that the solution is classical if either $n \leq 2$ or if $n \geq 3$ and the eigenvalues of the second matrix in (0.6) lie in an interval $\left(\lambda_{1}, \lambda_{2}\right)$ with $\lambda_{2}-\lambda_{1}$ small enough.

Our proof is based on establishing estimates on

$$
\begin{array}{ccc}
\|\nabla \ddot{u}\|_{L^{s}\left(Q_{T}\right)} & \text { (in } \S 2), \\
\left\|\nabla^{2} \dot{u}\right\|_{L_{2}\left(Q_{T}\right)} & \text { (in } \S 3), & \left.\left\|\nabla^{2} \dot{u}\right\|_{L^{s}\left(Q_{T}\right)} \quad \text { (in } \S 4\right)
\end{array}
$$

where $Q_{T}=\Omega \times(0, T)$, and finally on

$$
\left.\left\|\nabla^{2} \dot{u}\right\|_{L^{\infty}\left((0, T) ; L^{s}(\Omega)\right)} \quad \text { (in } \S 5\right),
$$

for some $s>2$, or for any $s>2$ if $\lambda_{2}-\lambda_{1}$ is small enough. 
Finally in $\S 6$ we consider the case where

$$
\sum_{i=1}^{n} \frac{\partial}{\partial x_{i}} \frac{\partial V(q)}{\partial q_{k i}}=\Delta \dot{u}_{k}
$$

but $W(p)$ is a general nonlinear function of $p$ (not necessarily isotropic) satisfying nonuniform ellipticity condition, and extend the result of Engler [3] by proving the existence of a strong solution for this case.

1. Existence and uniqueness. Let $N, n$ be positive integers and let $p=\left(p_{1}, \cdots, p_{N}\right), q=\left(q_{1}, \cdots, q_{N}\right)$ where $p_{k}=\left(p_{k 1}, \ldots, p_{k n}\right), q_{k}=$ $\left(p_{k 1}, \cdots, q_{k n}\right)$ are variable points in $\mathbf{R}^{n}$. We are given two functions

$$
W=W(p), \quad V=V(q) \text { from } \mathbf{R}^{n N} \text { into } \mathbf{R}^{1},
$$

satisfying the following conditions:

(1.2) $\tilde{\lambda}_{1}|\xi|^{2} \leq \sum_{k, l=1}^{N} \sum_{i, j=1}^{n} \frac{\partial^{2} W(p)}{\partial p_{k i} \partial p_{l j}} \xi_{i}^{k} \xi_{j}^{l} \leq \tilde{\lambda}_{2}|\xi|^{2} \quad\left(0<\tilde{\lambda}_{1}<\tilde{\lambda}_{2}<\infty\right)$,

$$
\lambda_{1}|\xi|^{2} \leq \sum_{k, l=1}^{N} \sum_{i, j=1}^{n} \frac{\partial^{2} V(q)}{\partial q_{k i} \partial q_{l j}} \xi_{i}^{k} \xi_{j}^{l} \leq \lambda_{2}|\xi|^{2} \quad\left(0<\lambda_{1}<\lambda_{2}<\infty\right)
$$

for all $p, q$ and $\mathbf{R}^{n N}$ and $\xi_{m}^{\lambda}$ real, where

$$
|\xi|^{2}=\sum_{k=1}^{N} \sum_{i=1}^{n}\left(\xi_{i}^{k}\right)^{2}
$$

Let $\Omega$ be a bounded domain in $\mathbf{R}^{n}$ with $C^{2}$ boundary $\partial \Omega$; for any $T>0$ we set

$$
\Omega_{T}=\{(x, T) ; x \in \Omega\}, \quad Q_{T}=\{(x, t) ; x \in \Omega, 0<t<T\} .
$$

We write $\partial / \partial t$ also as “.", i.e., $\dot{u}=\partial u / \partial t, \ddot{u}=\partial^{2} u / \partial t^{2}$, etc.

Consider the system of $N$ nonlinear partial differential equations

$$
\ddot{u}_{k}-\sum_{i=1}^{n} \frac{\partial}{\partial p_{k i}} \frac{\partial W(p)}{\partial p_{k i}}-\sum_{i=1}^{n} \frac{\partial}{\partial x_{i}} \frac{\partial V(q)}{\partial q_{k i}}=f_{k} \quad \text { in } Q_{T}
$$

where $p_{k}=\nabla u_{k}, q_{k}=\nabla \dot{u}_{k}\left(\right.$ thus $\left.p_{k i}=\partial u_{k} / \partial x_{i}, q_{k i}=\partial \dot{u}_{k} / \partial x_{i}\right)$, with initial conditions

$$
\begin{aligned}
& u_{k}(x, 0)=u_{k 0}(x), \\
& \dot{u}_{k}(x, 0)=u_{k 1}(x) \quad \text { for } x \in \Omega
\end{aligned}
$$


and boundary conditions

$$
u_{k}(x, t)=0 \text { for } x \in \partial \Omega, 0<t<T .
$$

We assume that

$$
\begin{gathered}
f_{k} \in L^{2}\left(Q_{T}\right) \quad \forall T>0, \\
u_{k 0}, u_{k 1} \text { belong to } W_{0}^{1,2}(\Omega) .
\end{gathered}
$$

Definition 1.1. A function $u=\left(u_{1}, \ldots, u_{N}\right)$ is called a weak solution of (1.4)-(1.6) if

$$
\begin{aligned}
& u \in C\left([0, T] ; W_{0}^{1,2}(\Omega)\right), \\
& \dot{u} \in L^{2}\left((0, T) ; W_{0}^{1,2}(\Omega)\right), \\
& \ddot{u} \in L^{2}\left((0, T) ; W^{-1,2}(\Omega)\right)
\end{aligned}
$$

where $W^{-1,2}(\Omega)$ is the conjugate of $W_{0}^{1,2}(\Omega)$, if (1.5) is satisfied (observe that $\dot{u} \in C\left([0, T] ; W^{-1,2}(\Omega)\right)$ and thus $\dot{u}_{k}(x, 0)$ is well defined in the trace class $W^{-1,2}(\Omega)$ ), and if (1.4) is satisfied in the following sense:

$$
\iint_{Q_{T}}\left[\ddot{u}_{k} \phi+\sum \frac{\partial W(p)}{\partial p_{k i}} \frac{\partial \phi}{\partial x_{i}}+\sum \frac{\partial V(q)}{\partial q_{k i}} \frac{\partial \phi}{\partial x_{i}}\right]=\iint_{Q_{T}} f_{k} \phi
$$

for any $\phi \in L^{2}\left((0, T) ; W_{0}^{1,2}(\Omega)\right)$.

Theorem 1.1. There exists a unique weak solution $u=\left(u_{1}, \ldots, u_{N}\right)$ of (1.4)-(1.6); further, if $u_{k 0}, u_{k 1}$ belong to $W^{2,2}(\Omega)$ then

$$
u \in C^{1}\left((0, T) ; W_{0}^{1,2}(\Omega)\right) \text {. }
$$

Indeed, existence and uniqueness have been established, for instance, in general Hilbert space framework in [5; Chapter 7, Theorem 1.2], and (1.9) follows from [5; Chapter 7, Theorem 3.2].

By taking a sequence $T_{m} \uparrow \infty$ and the corresponding solutions $u=$ $u_{T_{m}}$, and extracting a convergent subsequence, we obtain a solution $u$ of (1.4)-(1.6) for all $T>0$; by Theorem 1.1, the solution is unique.

We conclude this section with a conservation law. Multiplying (1.4) by $\dot{u}_{k}$, integrating over $Q_{t}$ and summing over $k$, we get

$$
\begin{aligned}
\frac{1}{2} \sum \int_{\Omega_{t}} \dot{u}_{k}^{2}-\frac{1}{2} \sum \int_{\Omega_{0}} \dot{u}_{k 0}^{2}+\iint_{Q_{t}} \sum \frac{\partial W(p)}{\partial p_{k i}} \frac{\partial p_{k i}}{\partial t} \\
+\iint_{Q_{t}} \sum \frac{\partial V(q)}{\partial q_{k i}} q_{k i}=\sum \iint_{Q_{t}} f_{k} \dot{u}_{k}
\end{aligned}
$$


and the third integral on the left-hand side is equal to

$$
\iint_{Q_{t}} \frac{d}{d t} W(p)=\int_{\Omega_{t}} W(p)-\int_{\Omega_{0}} W(p)
$$

Since, by (1.2), (1.3),

$$
\sum \frac{\partial V(q)}{\partial q_{k i}} q_{k i} \geq \frac{1}{2} \lambda_{1}|q|^{2}-C, \quad W(p) \geq \frac{1}{2} \tilde{\lambda}_{1}|p|^{2}-C,
$$

we obtain the estimate

$$
\int_{\Omega_{t}}|\dot{u}|^{2}+\int_{\Omega_{t}}|\nabla u|^{2}+\iint_{Q_{T}}|\nabla \dot{u}|^{2} \leq C \quad(C=C(T))
$$

for $0 \leq t \leq T$.

In $\S \S 2-5$ we assume that $W(p)$ and $V(q)$ belong to $C^{3}$,

$$
f_{k} \in W^{1, r}\left(Q_{T}\right) \quad \forall T>0, \quad 1<r<\infty,
$$

and derive additional regularity results for the weak solution (which already satisfies (1.8), (1.9) for all $T>0$ ); in particular, the solution will be shown to be classical in case $n \leq 2$.

2. $\nabla \ddot{u}$ is in $L^{s}$.

LEMMA 2.1. Suppose $v$ is a weak solution of

$$
\dot{v}-\gamma \Delta v=f_{0}+\sum_{i=1}^{n} \frac{\partial f i}{\partial x_{i}} \quad \text { in } Q_{T},
$$

$v=0$ on $\partial_{p} Q_{T}$, the parabolic boundary of $Q_{T}, f_{i} \in L^{q}\left(Q_{T}\right)$ for some $1<q<\infty(0 \leq i \leq n), 0<\gamma_{0} \leq \gamma \leq \gamma_{1}<\infty$ ( $\gamma, \gamma_{i}$ constants $)$. Then, for every $0<t<T$,

$$
\iint_{Q_{t}}\left\{\sum_{i=1}^{n}\left|\frac{\partial v}{\partial x_{i}}\right|^{q}\right\}^{1 / q} \leq C_{q} \iint_{Q_{i}}\left\{\sum_{i=0}^{n}\left|f_{i}\right|^{q}\right\}^{1 / q}
$$

where $C_{q}$ is a constant dependent only on $\Omega, \gamma_{0}, \gamma_{1}, T$ and $q$. of

Proof. Consider first the case $\Omega=\left\{x_{n}>0\right\}$. Let $u_{i}$ be the solutions

$$
\dot{u}_{i}-\gamma \Delta u_{i}=f_{i} \text { in } Q_{T} \text {, }
$$




$$
\begin{aligned}
u_{i}=0 & \text { on }\left\{x_{n}=0\right\} \times(0, T) \text { if } 0 \leq i \leq n-1, \\
\frac{\partial u_{n}}{\partial x_{n}}=0 & \text { on }\left\{x_{n}=0\right\} \times(0, T), \\
u_{i}(x, 0)=0 & \text { if } x \in \Omega, \quad 0 \leq i \leq n .
\end{aligned}
$$

Then

$$
v=u_{0}+\sum_{i=1}^{n} \frac{\partial u_{i}}{\partial x_{i}}
$$

Each $u_{i}(0 \leq i \leq n-1)$ can be represented by means of Green's function, and $u_{n}$ can be represented by means of Neumann's function. Applying $L^{q}$ parabolic estimates to the $u_{i}[9 ; \mathrm{IV}, \S 3]$ the assertion (2.1) follows. In order to extend (2.1) to general domains $\Omega$, we use partition of unity and proceed as in the derivation of $L^{q}$ estimates for parabolic equations in non-divergence form (see, e.g., [9]).

From now on we assume that (1.12)-(1.14) hold, and set

$$
A_{k l}^{i j}(q)=\frac{\partial^{2} V(q)}{\partial q_{k i} \partial q_{l j}}, \quad B_{k l}^{i j}(p)=\frac{\partial^{2} W(p)}{\partial p_{k i} \partial p_{l j}}
$$

THEOREM 2.2. For any $T>0$ there exist constants $p_{0}>2$ and $C>0$ such that

$$
\sum_{k=1}^{N}\left[\iint_{Q_{T}}\left|\nabla \ddot{u}_{k}\right|^{s}+\iint_{Q_{T}}\left|\nabla \dot{u}_{k}\right|^{s}\right] \leq C \quad \text { if } 2 \leq s \leq p_{0} .
$$

Proof. We first proceed formally, assuming that the solution is smooth. Let $z_{k}=\ddot{u}_{k}$. Differentiating (1.4) in $t$ we get

$$
\dot{z}_{k}-\sum_{i, j} \frac{\partial}{\partial x_{i}}\left(A_{k l}^{i j}(q) \frac{\partial z_{l}}{\partial x_{j}}\right)=\dot{f}_{k}-\sum_{i, j} \frac{\partial}{\partial x_{i}}\left(B_{k l}^{i j}(p) \frac{\partial \dot{u}_{l}}{\partial x_{j}}\right),
$$

and, by (1.12)-(1.14),

(2.5) $z_{k}(x, 0)$ belongs to $W_{0}^{1,2}(\Omega) \cap W^{1, r}(\Omega)$ for any $1<r<\infty$.

Let

$$
\gamma=\frac{\lambda_{1}+\lambda_{2}}{2}
$$

and rewrite (2.4) in the form

$$
\dot{z}_{k}-\gamma \Delta z_{k}=-\sum \frac{\partial}{\partial x_{i}}\left(\gamma \delta_{i j} \delta_{k l}-A_{k l}^{i j}(q)\right) \frac{\partial z_{l}}{\partial x_{j}}+G_{k}
$$


where

$$
G_{k}=\dot{f}_{k}-\sum \frac{\partial}{\partial x_{i}}\left(B_{k l}^{i j}(p) \frac{\partial \dot{u}_{l}}{\partial x_{j}}\right)
$$

In view of the choice (2.6), we have

$$
-\frac{\left(\lambda_{2}-\lambda_{1}\right)}{2}|\xi|^{2} \leq \sum\left(\gamma \delta_{i j} \delta_{k l}-A_{k l}^{i j}(q)\right) \xi_{i}^{k} \xi_{j}^{l} \leq \frac{\lambda_{2}-\lambda_{1}}{2}|\xi|^{2}
$$

where $|\xi|^{2}=\sum_{k, i}\left(\xi_{i}^{k}\right)^{2}$. Since $A_{k l}^{i j}=A_{l k}^{j i}$ by (2.2), the matrix $A_{\alpha, \beta} \equiv A_{k l}^{i j}$ where $\alpha=(i, k), \beta=(j, l)$ is symmetric, i.e., $A_{\alpha \beta}=A_{\beta \alpha}$. Hence (2.9) implies that for any real vectors $\xi=\left(\xi_{i}^{k}\right), \zeta=\left(\zeta_{i}^{k}\right)$,

$$
\left|\sum\left(\gamma \delta_{i j} \delta_{k l}-A_{k l}^{i j}(q) \xi_{i}^{k} \xi_{j}^{l}\right)\right| \leq \frac{1}{2}\left(\lambda_{2}-\lambda_{1}\right)|\xi||\zeta| .
$$

Consider the linear parabolic system

$$
\begin{gathered}
\dot{z}_{k}-\gamma \Delta z_{k}=-\sum \frac{\partial}{\partial x_{i}}\left[\left(\gamma \delta_{i j} \delta_{k l}-A_{k l}^{i j}(q)\right) h_{l j}\right] \\
+\sum \frac{\partial}{\partial x_{j}} g_{k j}+g_{k 0} \quad \text { in } Q_{T} \\
z_{k}=0 \text { on } \partial_{p} Q_{T}
\end{gathered}
$$

where $q=\nabla \dot{u}$, and $G \equiv\left(h_{l j}, g_{k i}\right)(1 \leq l, k \leq N$ and $1 \leq j \leq n$, $0 \leq i \leq n)$ is any vector with components in $L^{s}\left(Q_{T}\right)$. We introduce the norm

$$
\|G\|_{s}=\left\{\iint_{Q_{T}}\left[\sum\left|h_{l j}\right|^{s}+M \sum\left|g_{k i}\right|^{s}\right]\right\}^{1 / s}
$$

where $M$ is a positive constant to be determined.

For any $G$, there is a unique solution $z=\left(z_{1}, \ldots, z_{N}\right)$ of (2.11), (2.12). We denote the vector $\left(\nabla z_{1}, \ldots, \nabla z_{N}\right)$ by $\nabla z$ and set $\nabla z=S G$; we also define the norm

$$
\|\nabla z\|_{s}=\left\{\iint_{Q_{T}} \sum\left|\nabla z_{k}\right|^{s}\right\}^{1 / s} .
$$

Then $S$ is a linear mapping from $L^{s}$ into $L^{s}$ (with the norms defined by (2.13), (2.14)), and by Lemma 2.1

$$
\|S\|_{s} \leq A_{s}<\infty
$$

where $A_{s}$ is a constant depending on $s$, as well as on $\lambda_{1}, \lambda_{2}, T$ and $\Omega$. We claim that

$$
\|S\|_{2} \leq \frac{\lambda_{2}-\lambda_{1}}{\lambda_{2}+\lambda_{1}}<1
$$


Indeed, if we multiply (2.11) by $z_{k}$ and integrate over $\Omega_{t}$, we get, after summing on $k$,

$$
\begin{aligned}
\int_{\Omega_{T}} \sum\left|z_{k}\right|^{2}+\gamma \iint_{Q_{T}} \sum\left|\nabla z_{k}\right|^{2} \\
=\iint_{Q_{T}} \sum\left(\gamma \delta_{i j} \delta_{k l}-A_{k l}^{i j}(q)\right) h_{l j} \frac{\partial z_{k}}{\partial x_{i}} \\
\quad-\iint_{Q_{T}}\left(\sum_{k=1}^{N} \sum_{j=1}^{n} g_{k j} \frac{\partial z_{k}}{\partial x_{j}}\right)+\iint_{Q_{T}} \sum g_{k 0} z_{k} .
\end{aligned}
$$

In view of $(2.10)$, the first integral on the right-hand side is bounded by

$$
\begin{aligned}
\frac{1}{2}\left(\lambda_{2}-\lambda_{2}\right) \iint_{Q_{T}}\left(\sum\left|h_{l j}\right|^{2}\right)^{1 / 2}\left(\sum\left|\frac{\partial z_{k}}{\partial x_{i}}\right|^{2}\right)^{1 / 2} \\
=\frac{1}{4}\left(\lambda_{2}-\lambda_{1}\right)\left\{\iint_{Q_{T}} \sum\left|h_{l j}\right|^{2}+\iint_{Q_{T}}\left|\nabla z_{k}\right|^{2}\right\} .
\end{aligned}
$$

Recalling (2.6) we obtain

$$
\begin{aligned}
& \iint_{Q_{T}}\left(\sum\left|\nabla z_{k}\right|^{2}\right) \\
& \leq \frac{\lambda_{2}-\lambda_{1}}{\lambda_{2}+3 \lambda_{1}} \iint_{Q_{T}}\left(\sum\left|h_{l j}\right|^{2}\right) \\
&+C {\left[\iint_{Q_{T}}\left(\sum_{k=1}^{N} \sum_{j=1}^{n}\left|g_{k j}\right|^{2}\right]^{2}\left[\iint_{Q_{T}} \sum\left|\nabla z_{k}\right|^{2}\right]^{1 / 2}\right.} \\
&+C {\left[\iint_{Q_{T}} \sum\left|g_{k 0}\right|^{2}\right]^{1 / 2}\left[\iint_{Q_{T}} \sum z_{k}^{2}\right]^{1 / 2} . }
\end{aligned}
$$

Since

$$
\iint_{Q_{T}}\left|z_{k}\right|^{2} \leq C \iint_{Q_{T}}\left|\nabla z_{k}\right|^{2}
$$

the assertion (2.16) now follows provided $M$ is chosen sufficiently large (depending on the constant $C$ in (2.17)).

By the Riesz-Thorin theorem, $\log \|S\|_{1 / \beta}$ is a convex function of $\beta$, $0<\beta<1$. Hence,

$$
\|S\|_{s} \leq\|S\|_{2}^{\alpha}\|S\|_{q_{0}}^{1-\alpha} \quad \text { if } \frac{1}{s}=\frac{1-\alpha}{q_{0}}+\frac{\alpha}{2}, \quad q_{0}>2, \quad 0 \leq \alpha \leq 1 .
$$


Using (2.15), (2.16) we conclude that

$$
\|S\|_{s} \leq\left(\frac{\lambda_{2}-\lambda_{1}}{\lambda_{2}+\lambda_{1}}\right)^{\alpha}\left(A_{q_{0}}\right)^{1-\alpha} \equiv \vartheta_{s} \quad \text { if } \frac{1}{s}=\frac{1-\alpha}{q_{0}}+\frac{\alpha}{2} ;
$$

notice that $\vartheta_{s}<1$ if $s-2$ is small enough.

In view of $(2.7),(2.8)$, the functions $\tilde{z}_{k}(x, t) \equiv z_{k}(x, t)-z_{k}(x, 0)$ satisfy a system of the form (2.11), (2.12) with

$$
\begin{gathered}
h_{l j}=\frac{\partial \tilde{z}_{l}}{\partial x_{j}}, \quad g_{k j}=-\sum B_{k l}^{i j}(p) \frac{\partial \dot{u}_{l}}{\partial x_{j}} \quad(j>0), \\
g_{k 0}=\dot{f}_{k}+\tilde{g}_{k}
\end{gathered}
$$

and $\tilde{g}_{k}$ depending on the data $z_{i}(x, 0)$. By $(2.18)$,

$$
\begin{aligned}
\iint_{Q_{t}}|\nabla \tilde{z}|^{s} & \leq\left(\|S\|_{s}\right)^{s} \iint_{Q_{t}}\left\{\sum\left|h_{l j}\right|^{s}+M \sum\left|g_{k i}\right|^{s}\right\} \\
& \leq\left(\vartheta_{s}\right)^{s} \iint_{Q_{t}}|\nabla \tilde{z}|^{s}+\left(\vartheta_{s}\right)^{s} M \iint_{Q_{t}} \sum\left|g_{k i}\right|^{s}
\end{aligned}
$$

where $\vartheta_{s}<1$. Hence

$$
\iint_{Q_{t}}|\nabla \tilde{z}|^{s} \leq \frac{M}{1-\left(\vartheta_{s}\right)^{s}} \iint_{Q_{t}} \sum\left|g_{k i}\right|^{s} .
$$

Recalling that $z_{k}=\ddot{u}_{k}$, we conclude that

$$
\iint_{Q_{t}} \sum\left|\nabla \ddot{u}_{k}\right|^{s} \leq C+C \iint_{Q_{i}} \sum\left|\nabla \dot{u}_{k}\right|^{s}
$$

provided $t=T$ and $s$ is such that $\vartheta_{s}<1$; this estimate is valid also for any $t \in(0, T)$, since $A_{q_{0}}$ and $\vartheta_{s}$ are independent of $t$.

Set

$$
\phi(t)=\iint_{Q_{t}} \sum\left|\nabla \ddot{u}_{k}\right|^{s}
$$

Since

$$
\nabla \dot{u}_{k}(x, t)=\nabla \dot{u}_{k}(x, 0)+\int_{0}^{t} \nabla \ddot{u}_{k}(x, \tau) d \tau
$$

we have

$$
\iint_{Q_{t}}\left|\nabla \dot{u}_{k}\right|^{s} \leq C+C \int_{0}^{t} \phi(\tau) d \tau
$$

Substituting this into (2.19), we get

$$
\phi(t) \leq C+C \int_{0}^{t} \phi(\tau) d \tau
$$

Hence, by Gronwall's inequality, $\phi(t) \leq C$, and (2.3) follows. 
So far we have assumed that the solution was smooth enough. In order to prove (2.3) rigorously, we approximate the initial values by smooth initial values, say $u_{k 0}^{\lambda}, u_{k 1}^{\lambda}(\lambda \rightarrow 0)$ in $C_{0}^{2}(\Omega)$. By a fixed point argument one can prove that (1.4)-(1.6) with initial data $u_{k 0}^{\lambda}, u_{k 1}^{\lambda}$ has a classical solution in $Q_{\sigma}$ if $\sigma=\sigma_{\lambda}$ is small enough; $\sigma_{\lambda} \rightarrow 0$ if $\lambda \rightarrow 0$. The unique weak solution $u_{k}^{\lambda}$ asserted in Theorem 1.1 which corresponds to the data $u_{k 0}^{\lambda}, u_{k 1}^{\lambda}$ must coincide with the classical solution for $t<\sigma_{\lambda}$, and, as $\lambda \rightarrow 0$,

$$
u_{k}^{\lambda} \rightarrow u_{k} \quad \text { in } C^{1}\left([0, T] ; W_{0}^{1,2}(\Omega)\right) .
$$

We now work with finite differences in time; that is, setting

$$
z_{k}(x, t)=\Delta_{h} \dot{u}_{k}(x, t) \equiv \frac{\dot{u}_{k}(x, t+h)-\dot{u}_{k}(x, t)}{h}
$$

and finite-differencing (1.4) in time, we obtain a system similar to (2.4) with $A_{k l}^{i j}$ replaced by

$$
\tilde{A}_{k l}^{i j}=\int_{0}^{1} \frac{\partial^{2} V}{\partial q_{k i} \partial q_{l j}}(\nabla \dot{u}(x, t)+\tau(\nabla \dot{u}(x, t+h)-\nabla \dot{u}(x, t))) d \tau
$$

and similarly $B_{k l}^{i j}$ replaced by $\tilde{B}_{k l}^{i j}$ and $\dot{f}_{k}$ replaced by $\Delta_{h} f_{k}$. Since $u_{k}^{\lambda}$ is smooth in $\bar{Q}_{\sigma \lambda},(2.5)$ is satisfied.

Proceeding as before, we deduce that

$$
\sum \iint_{Q_{T}}\left|\Delta_{h}\left(\nabla \dot{u}_{k}^{\lambda}\right)\right|^{s} \leq C+C \sum \int_{\Omega}\left|\Delta_{h} \nabla \dot{u}_{k}^{\lambda}(x, 0)\right|^{s} .
$$

As $h \rightarrow 0$ the right-hand side converges to

$$
C+C \sum \int_{\Omega}\left|\nabla \ddot{u}_{k}^{\lambda}(x, 0)\right|^{s} \text {. }
$$

Since, in view of (1.14), we can choose the approximations $u_{k 0}^{\lambda}, u_{k l}^{\lambda}$ such that the right-hand side of (2.22) is bounded independently of $\lambda$, it follows from (2.21) that

$$
\sum \iint_{Q_{T}}\left|\nabla \ddot{u}_{k}^{\lambda}\right|^{s} \leq C,
$$

where $C$ is a constant independent of $\lambda$. Letting $\lambda \rightarrow 0$ and recalling (2.20), the assertion (2.3) follows.

The proof of Theorem 2.2 yields:

Corollary 2.3. For any $2<p_{0}<q_{0}$, if $\lambda_{2}-\lambda_{1}$ is sufficiently small so that $\vartheta_{p_{0}}<1\left(\vartheta_{p_{0}}\right.$ as in (2.18)), then (2.3) holds for all $2 \leq s \leq p_{0}$. 
3. $\nabla^{2} u$ is in $L^{2}$.

LEMMA 3.1. There exists a constant $C$ such that

$$
\sum_{k=1}^{N}\left\{\iint_{Q_{T}}\left|\nabla^{2} \dot{u}_{k}\right|^{2}+\iint_{Q_{T}}\left|\nabla^{2} u_{k}\right|^{2}\right\} \leq C .
$$

Proof. We first establish that $\nabla^{2} u$ and $\nabla^{2} \dot{u}$ belong to $L^{2}\left(\Omega_{0} \times(0, T)\right)$ where $\Omega_{0}$ is any compact subdomain of $\Omega$. We shall begin be deriving a priori estimates, assuming for the moment that $\nabla^{2} u$ and $\nabla^{2} \dot{u}$ are indeed in $L^{2}$.

Let $B_{R}$ and $B_{R^{\prime}}$, be two concentric balls with radii $R$ and $R^{\prime}$ respectively, $R<R^{\prime}$, and $\overline{B_{R^{\prime}}} \subset \Omega$. Let $\eta$ be a cut-off function: $\eta \in C_{0}^{\infty}\left(B_{R^{\prime}}\right)$, $\eta=1$ in $B_{R}$. Fix any integer $m, 1 \leq m \leq n$, and set $z_{k}=\partial \dot{u}_{k} / \partial x_{m}$. Differentiating (1.4) with respect to $x_{m}$ we get

(3.2) $\dot{z}_{k}-\sum \frac{\partial}{\partial x_{i}}\left(A_{k l}^{i j}(q) \frac{\partial z_{l}}{\partial x_{j}}\right)=\frac{\partial f_{k}}{\partial x_{m}}+\sum \frac{\partial}{\partial x_{i}}\left(B_{k l}^{i j}(p) \frac{\partial}{\partial x_{j}} \frac{\partial u_{l}}{\partial x_{m}}\right)$.

The functions $Z_{k}=\eta z_{k}$ satisfy:

$$
\begin{aligned}
\dot{Z}_{k} & -\sum_{i, j, l} \frac{\partial}{\partial x_{j}}\left(A_{k l}^{i j}(q) \frac{\partial Z_{l}}{\partial x_{j}}\right) \\
& =\sum_{i, j, l} \frac{\partial}{\partial x_{i}}\left(B_{k l}^{i j}(p) \frac{\partial}{\partial x_{j}}\left(\eta \frac{\partial u_{l}}{\partial x_{m}}\right)\right)+F_{k}
\end{aligned}
$$

where

$$
\begin{aligned}
F_{k}= & \eta \frac{\partial F_{k}}{\partial x_{m}}-\sum \frac{\eta_{x_{i}}}{\eta} A_{k l}^{i j} \frac{\partial Z_{l}}{\partial x_{j}} \\
& -\sum \frac{\partial}{\partial x_{i}}\left(A_{k l}^{i j} \eta_{x_{j}} z_{l}\right) \sum \frac{\eta_{x_{i}} \eta_{x_{j}}}{\eta} A_{k l}^{i j} z_{l} \\
& -\sum \frac{\eta_{x_{i}}}{\eta} B_{k l}^{i j} \frac{\partial}{\partial x_{j}}\left(\eta \frac{\partial u_{l}}{\partial x_{m}}\right)-\sum \frac{\partial}{\partial x_{i}}\left(B_{k l}^{i j} \eta_{x_{j}} \frac{\partial u_{l}}{\partial x_{m}}\right) \\
& +\sum \frac{\eta_{x_{i}} \eta_{x_{j}}}{\eta} B_{k l}^{i j} \frac{\partial u_{l}}{\partial x_{m}}
\end{aligned}
$$

Multiplying (3.3) by $Z_{k}$ and integrating over $Q_{t}$, we get, after summing over $k$,

$$
\begin{aligned}
& \frac{1}{2} \sum \int_{\Omega_{t}} Z_{k}^{2}-\frac{1}{2} \sum \int_{\Omega_{0}} Z_{k}^{2}+\sum \iint_{Q_{t}} A_{k l}^{i j} \frac{\partial Z_{l}}{\partial x_{j}} \frac{\partial Z_{k}}{\partial x_{i}} \\
& =\sum \iint_{Q_{t}} B_{k l}^{i j} \frac{\partial}{\partial x_{j}}\left(\eta \frac{\partial u_{l}}{\partial x_{m}}\right) \frac{\partial Z_{k}}{\partial x_{j}}+\sum \iint_{Q_{t}} F_{k} Z_{k}
\end{aligned}
$$


Recalling that $Z_{k}=\eta z_{k}$ and using (1.11) and (3.4), we easily estimate $\left|\sum \iint_{Q_{t}} F_{k} Z_{k}\right| \leq C+C \sum \iint_{Q_{t}}|\nabla Z||z|+C \sum \iint_{Q_{t}}\left|\nabla\left(\eta \frac{\partial u_{k}}{\partial x_{m}}\right)\right||z|$ where $|z|^{2}=\sum\left|z_{k}\right|^{2},|\nabla Z|^{2}=\sum\left|\nabla Z_{k}\right|^{2}$. Hence

$$
\begin{aligned}
& \left|\sum \iint_{Q_{t}} F_{k} Z_{k}\right| \\
& \quad \leq C+C\left[\iint_{Q_{t}}|\nabla z|^{2}\right]^{1 / 2}+C\left[\sum_{k=1}^{N} \iint_{Q_{t}}\left|\nabla\left(\eta \frac{\partial u_{k}}{\partial x_{m}}\right)\right|^{2}\right]^{1 / 2} .
\end{aligned}
$$

Substituting this into (3.5) and using (1.2), (1.3), we easily obtain, after using the Schwarz inequality,

$$
\sum \iint_{Q_{t}}\left|\nabla Z_{k}\right|^{2} \leq C+\sum \iint_{Q_{t}}\left|\nabla\left(\eta \frac{\partial u_{k}}{\partial x_{m}}\right)\right|^{2} \text {. }
$$

Since

$$
Z_{k}=\eta \frac{\partial \dot{u}_{k}}{\partial x_{m}}=\frac{\partial}{\partial t}\left(\frac{\partial u_{k}}{\partial x_{m}}\right)
$$

we have

$$
\begin{aligned}
\left|\nabla\left(\eta \frac{\partial u_{k}}{\partial x_{m}}\right)\right|^{2} & =\left|\nabla\left(\eta \frac{\partial u_{k 0}}{\partial x_{m}}\right)+\int_{0}^{t} Z_{k}(x, s) d s\right|^{2} \\
& \leq 2\left|\nabla\left(\eta \frac{\partial u_{k 0}}{\partial x_{m}}\right)\right|^{2}+2 T \int_{0}^{t}\left|Z_{k}(x, s)\right|^{2} d s .
\end{aligned}
$$

Substituting this into (3.7) and setting

$$
\phi(t)=\sum \iint_{Q_{t}}\left|\nabla Z_{k}\right|^{2}
$$

we obtain

$$
\phi(t) \leq C+C \int_{0}^{t} \phi(s) d s .
$$

Hence, by Gronwall's inequality,

$$
\sum_{k} \iint_{Q_{T}}\left|\nabla\left(\eta \frac{\partial \dot{u}_{k}}{\partial x_{m}}\right)\right|^{2} \leq C,
$$

and then also

$$
\sum_{k} \iint_{Q_{T}}\left|\nabla\left(\eta \frac{\partial u_{k}}{\partial x_{m}}\right)\right|^{2} \leq C
$$


In order to prove (3.8), (3.9) rigorously, we work with finite differences, replacing $\partial u_{k} / \partial x_{m}$ by

$$
\left(\Delta_{m}^{k} u_{k}\right)(x, t)=\frac{u_{k}\left(x+h e_{m}, t\right)-u_{k}(x, t)}{h}
$$

where $e_{m}$ is the unit vector in the $x_{m}$-direction. Then, with $z_{k}=$ $\Delta_{m}^{h} \dot{u}_{k}, Z_{k}=\eta z_{k}$, the relations (3.2)-(3.4) hold with minor differences, namely, $A_{k l}^{i j}(p)$ is replaced by

$$
\tilde{A}_{k l}^{i j}=\int_{0}^{1} \frac{\partial^{2} V}{\partial q_{k i} \partial q_{l j}}\left(\nabla \dot{u}(x, t)+\tau\left(x+h e_{m}, t\right)-\nabla \dot{u}(x, t)\right) d \tau
$$

and similarly for $B_{k l}^{i j}$, and $\partial f_{k} / \partial x_{m}$ is replaced by $\Delta_{m}^{h} f_{k}$; notice that $\nabla z_{k}$ and $\nabla \dot{u}_{k}$ belong to $L^{2}\left(Q_{T}\right)$ (by Theorem 2.2). We can now proceed as before (but this time rigorously), to establish analogously to (3.8), (3.9), that

$$
\sum_{k}\left[\iint_{Q_{T}}\left|\nabla\left(\eta \Delta_{m}^{h} \dot{u}_{k}\right)\right|^{2}+\iint_{Q_{T}}\left|\nabla\left(\eta \Delta_{m}^{h} u_{k}\right)\right|^{2}\right] \leq C ;
$$

$C$ is independent of $h$. By standard lemma in calculus it follows that, in $B_{R} \times(0, T)$ (where $\left.\eta=1\right)$, the derivatives

$$
\nabla \frac{\partial \dot{u}_{k}}{\partial x_{m}}, \quad \nabla \frac{\partial u_{k}}{\partial x_{m}}
$$

exist and belong to $L^{2}\left(B_{R} \times(0, T)\right)$, and

$$
\sum_{k}\left[\int_{0}^{T} \int_{B_{R}}\left|\nabla \frac{\partial \dot{u}_{k}}{\partial x_{m}}\right|^{2}+\int_{0}^{T} \int_{B_{R}}\left|\nabla \frac{\partial u_{k}}{\partial x_{m}}\right|^{2}\right] \leq C
$$

this holds for every $1 \leq m \leq n$.

We next proceed to extend the interior estimates (3.11) to the boundary, replacing $B_{R}$ by $B_{R}\left(x_{0}\right) \cap \Omega$ where $B_{R}\left(x_{0}\right)=\left\{x \in \mathbf{R}^{n},\left|x-x_{0}\right|<\right.$ $R\}$ and $x_{0}$ is any point on $\partial \Omega$. Suppose $\partial \Omega \cap B_{R_{0}}\left(x_{0}\right)$ (for some $\left.0<R<R_{0}\right)$ is given by $x_{n}=h\left(x_{1}, \ldots, x_{n-1}\right)$ with $h \in C^{2}$, such that $x_{n}>h$ in $\Omega \cap B_{R_{0}}\left(x_{0}\right)$. Take for simplicity $x_{0}=(0, \ldots, 0)$ and introduce new variables

$$
\begin{aligned}
& x_{i}^{\prime}=x_{i} \quad \text { if } 1 \leq i \leq n-1, \\
& x_{n}^{\prime}=x_{n}-h\left(x_{1}, \ldots, x_{n-1}\right) ;
\end{aligned}
$$

it will be convenient to write $x_{i}^{\prime}=h_{i}(x)$. Setting

$$
U_{k}\left(x^{\prime}, t\right)=u_{k}(x, t) \text {, }
$$


(1.4) becomes

$$
\ddot{U}_{k}-\sum_{\lambda=1}^{n} \frac{\partial}{\partial x_{\lambda}^{\prime}}\left(\frac{\partial h_{\lambda}}{\partial x_{i}} \frac{\partial W(P)}{\partial p_{k i}}\right)-\sum_{\lambda=1}^{n} \frac{\partial}{\partial x_{\lambda}^{\prime}}\left(\frac{\partial h_{\lambda}}{\partial x_{i}} \frac{\partial V(Q)}{\partial q_{k i}}\right)=f_{k}
$$

where $P=\left(P_{1}, \ldots, P_{N}\right), Q=\left(Q_{1}, \ldots, Q_{N}\right), P_{k}=\left(P_{k 1}, \ldots, P_{k n}\right), Q_{k}=$ $\left(Q_{k 1}, \ldots, Q_{k n}\right)$, and

$$
P_{k i}=\sum_{\mu=1}^{n} \frac{\partial U_{k}}{\partial x_{\mu}^{\prime}} \frac{\partial h_{\mu}}{\partial x_{i}}, \quad Q_{k i}=\sum_{\mu=1}^{n} \frac{\partial \dot{U}_{k}}{\partial x_{\mu}^{\prime}} \frac{\partial h_{\mu}}{\partial x_{i}}
$$

As before we first proceed formally, differentiating (3.11) in any tangential direction $x_{m}(1 \leq m \leq n-1)$. Setting $z_{k}=\partial \dot{U} / \partial x_{m}$, $Z_{k}=\eta z_{k}$ where $\eta\left(x^{\prime}\right)$ is a cut-off function $\eta \in C_{0}^{\infty}\left(B_{\rho^{\prime}}\right), \eta=1$ in $B_{\rho}$ for some $0<\rho<\rho^{\prime}$, and defining

$$
\tilde{A}_{k l}^{\lambda \mu}=\sum_{i, j=1}^{n} A_{k l}^{i j} \frac{\partial h_{\lambda}}{\partial x_{i}} \frac{\partial h_{\mu}}{\partial x_{j}}, \quad \tilde{B}_{k l}^{\lambda \mu}=\sum_{i, j=1}^{n} B_{k l}^{i j} \frac{\partial h_{\lambda}}{\partial x_{i}} \frac{\partial h_{\mu}}{\partial x_{j}},
$$

we find that

$$
\begin{aligned}
\dot{Z}_{k} & -\sum_{i, j} \frac{\partial}{\partial x_{i}^{\prime}}\left(\tilde{A}_{k l}^{i j}(Q) \frac{\partial Z_{l}}{\partial x_{j}^{\prime}}\right) \\
& =\sum_{i, j} \frac{\partial}{\partial x_{i}^{\prime}}\left(\tilde{B}_{k l}^{i j}(P) \frac{\partial}{\partial x_{j}^{\prime}}\left(\eta \frac{\partial U_{l}}{\partial x_{m}^{\prime}}\right)\right)+\tilde{F}_{k}
\end{aligned}
$$

where $\tilde{F}_{k}$ is defined similarly to $F_{k}$ in (3.4); the difference is that $A_{k l}^{i j}, B_{k l}^{i j}$ are replaced by $\tilde{A}_{k l}^{i j}, \tilde{B}_{k l}^{i j}$, that $u_{l}$ is replaced by $U_{l}$, and that the variables $x_{j}$ are replaced by the variables $x_{j}^{\prime}$.

Multiplying (3.13) by $Z_{k}$ and integrating over $(x, t)$, and proceeding as before, we arrive at the analogs of (3.8), (3.9). If we work with finite differences $\Delta_{m}^{h}$ (instead of with $\partial / \partial x_{m}$ ), then we can establish rigorously the analogs of (3.10) and (3.11); thus

$$
\nabla \frac{\partial}{\partial x_{m}^{\prime}} \dot{U}_{k} \text { and } \nabla \frac{\partial}{\partial x_{m}^{\prime}} U_{k}
$$

belong to $L^{2}\left(B_{\rho} \times(0, T)\right)$ and

$$
\sum_{k} \int_{0}^{t} \int_{B_{p}}\left[\left|\nabla \frac{\partial}{\partial x_{m}^{\prime}} \dot{U}_{k}\right|^{2}+\left|\nabla \frac{\partial}{\partial x_{m}^{\prime}} U_{k}\right|^{2}\right] \leq C
$$

$$
(1 \leq m \leq n-1)
$$


It remains to consider the normal derivatives $\partial\left(\nabla \dot{U}_{k}\right) / \partial x_{n}^{\prime}$, $\partial\left(\nabla U_{k}\right) / \partial x_{n}^{\prime}$. Since we have already proved that $\nabla^{2} \dot{u}_{k}, \nabla^{2} u_{k}, \nabla^{2} u_{k}$ are locally in $L^{2}\left(Q_{T}\right)$, the same is true of $\nabla^{2} \dot{U}_{k}, \nabla^{2} U_{k}$. Hence we can apply the $\partial / \partial x_{\lambda}^{\prime}$ differentiation to $\partial W / \partial p_{k i}, \partial V / \partial q_{k i}$ in (3.12), thus obtaining the relation

$$
\ddot{U}_{k}-\sum \tilde{B}_{k l}^{i j} \frac{\partial^{2} \dot{U}_{l}}{\partial x_{i}^{\prime} \partial x_{j}^{\prime}}-\sum \tilde{A}_{k l}^{i j} \frac{\partial^{2} U_{l}}{\partial x_{i}^{\prime} \partial x_{j}^{\prime}}=f_{k} \quad \text { in } B_{\rho} \times(0, T) \text {. }
$$

In view of Theorem 2.2 and (3.14), we can estimate the $L^{2}\left(B_{\rho} \times(0, T)\right)$ norm of $\ddot{U}_{k}$ and of

$$
\frac{\partial^{2} \dot{U}_{l}}{\partial x_{i}^{\prime} \partial x_{j}^{\prime}}, \quad \frac{\partial^{2} U_{l}}{\partial x_{i}^{\prime} \partial x_{j}^{\prime}} \quad \text { for all }(i, j) \neq(n, n) .
$$

It follows that

$$
\sum_{l} \tilde{B}_{k l}^{n n} \frac{\partial^{2} \dot{U}_{l}}{\left.\partial\left(x_{n}^{\prime}\right)^{2}\right)}+\sum_{l} \tilde{A}_{k l}^{n n} \frac{\partial^{2} U_{l}}{\partial\left(x_{n}^{\prime}\right)^{2}}=\tilde{g}_{k},
$$

where

$$
\sum \int_{0}^{T} \int_{B_{p}} \tilde{g}_{k}^{2} \leq C
$$

By ellipticity, the matrix $\left(\tilde{B}_{k l}^{n n}\right)_{k, l=1}^{N}$ is uniformly positive. Hence we can solve from (3.15),

$$
\frac{\partial^{2} \dot{U}_{k}}{\partial\left(x_{n}^{\prime}\right)^{2}}=\sum_{l} a_{k l} \frac{\partial^{2} U_{l}}{\partial\left(x_{n}^{\prime}\right)^{2}}+\tilde{g}_{k}
$$

where $\tilde{g}_{k}$ is another function, still satisfying (3.16), and $a_{k l}$ are uniformly bounded functions. Setting

$$
\phi(t)=\sum_{k} \int_{0}^{t} \int_{B_{p}}\left|\frac{\partial^{2} \dot{U}_{k}}{\partial\left(x_{n}^{\prime}\right)^{2}}\right|^{2}
$$

we easily deduce from (3.17) that

$$
\phi(t) \leq C+C^{t} \int_{0} \phi(s) d s
$$

and therefore

$$
\sum_{k} \int_{0}^{T} \int_{B_{\rho}}\left[\left|\frac{\partial^{2} \dot{U}_{k}}{\partial\left(x_{n}^{\prime}\right)^{2}}\right|^{2}+\left|\frac{\partial^{2} U_{k}}{\partial\left(x_{n}^{\prime}\right)^{2}}\right|^{2}\right] \leq C .
$$


Combining this with (3.14) we find, after going back to the variables $x_{i}$, that (3.11) holds with $B_{R}$ replaced by $B_{R}\left(x_{0}\right) \cap \Omega$ (for $R$ small enough), for all $1 \leq m \leq n$. This completes the proof of Theorem 3.1.

4. $\nabla^{2} \dot{u}$ is in $L^{s}$. In this section we prove:

THEOREM 4.1. For any $T>0$ there exist constants $p_{0}>2$ and $C>0$ such that

$$
\sum_{k=1}^{N}\left[\iint_{Q_{T}}\left|\nabla^{2} \dot{u}_{k}\right|^{s}+\iint_{Q_{T}}\left|\nabla^{2} u_{k}\right|^{s}\right] \leq C \quad \text { if } 2 \leq s \leq p_{0} .
$$

Proof. We first establish the assertion of the theorem in $\Omega_{0} \times(0, T)$ where $\Omega_{0}$ is any compact subdomain of $\Omega$. We begin by taking any two concentric balls $B_{R}, B_{R^{\prime}}$ with $R<R^{\prime}, \overline{B_{R^{\prime}}} \subset \Omega$ as in the proof of Theorem 3.1, and introducing the functions $z_{k}=\partial \dot{u}_{k} / \partial x_{m}, Z_{k}=\eta z_{k}$ as before. We assume for the moment that $\nabla z_{k}$ is in $L^{S}\left(Q_{T}\right)$. By (3.3), (3.4) we have,

$$
\dot{Z}_{k}-\gamma \Delta Z_{k}=-\sum_{i, j, l} \frac{\partial}{\partial x_{i}}\left(\left(\gamma \delta_{i j} \delta_{k l}-A_{k l}^{i j}(q)\right) \frac{\partial Z_{l}}{\partial x_{j}}\right)+G_{k}
$$

where $\gamma$ is as in (2.6) and

$$
\begin{aligned}
G_{k}= & \sum \frac{\partial}{\partial x_{j}}\left(B_{k l}^{i j}(p) \frac{\partial}{\partial x_{j}}\left(\eta \frac{\partial u_{l}}{\partial x_{m}}\right)\right)+\eta \frac{\partial f_{k}}{\partial x_{m}} \\
& -\sum \eta_{x_{l}} A_{k l}^{i j} \frac{\partial z_{l}}{\partial x_{j}}-\sum \frac{\partial}{\partial x_{i}}\left(A_{k l}^{i j} \eta_{x_{j}} z_{l}\right) \\
& -\sum \eta_{x_{i}} B_{k l}^{i j} \frac{\partial}{\partial x_{j}} \frac{\partial u_{l}}{\partial x_{m}}-\sum \frac{\partial}{\partial x_{i}}\left(B_{k l}^{i j} \eta_{x_{j}} \frac{\partial u_{l}}{\partial x_{m}}\right)
\end{aligned}
$$

Let

$$
\tilde{p}=\frac{n}{n-2} \quad \text { if } n \geq 3, \quad \tilde{p}=\infty \quad \text { if } n \leq 2
$$

Set

$$
\text { (4.4) } \begin{aligned}
h_{k} & =\eta \frac{\partial f_{k}}{\partial x_{m}}-\sum_{i, j, l} \eta_{x_{i}} A_{k l}^{i j}(q) \frac{\partial z_{l}}{\partial x_{j}}-\sum_{i, j, l} \eta_{x_{i}} B_{k l}^{i j}(p) \frac{\partial}{\partial x_{j}} \frac{\partial u_{l}}{\partial x_{m}} \\
g_{j k, 1} & =-\sum_{i, l} A_{k l}^{i j}(q) \eta_{x_{j}} z_{l}-\sum_{i, l} B_{k l}^{i j}(p) \eta_{x_{j}} \frac{\partial u_{l}}{\partial x_{m}}
\end{aligned}
$$

By Theorem 3.1 and Sobolev's imbedding,

$$
\iint_{Q_{T}} h_{k}^{2} \leq C, \quad \iint_{Q_{T}}\left|g_{j k, 1}\right|^{r} \leq C \quad \forall r<\tilde{p}
$$


Let $U_{k}$ and $V_{k}$ be the solutions of

$$
\begin{aligned}
& \dot{U}_{k}-\gamma \Delta U_{k}=h_{k} \text { in } Q_{T}, \\
& U_{k}=Z_{k}(x, 0) \text { on } \partial_{p} Q_{T},
\end{aligned}
$$

and

$$
\begin{aligned}
\dot{V}_{k}-\gamma \Delta V_{k} & =\sum \frac{\partial}{\partial x_{j}} g_{j k, 1} \quad \text { in } Q_{T}, \\
V_{k} & =0 \quad \text { on } \partial_{p} Q_{T} .
\end{aligned}
$$

By $L^{2}$ estimates for the heat operator,

$$
\begin{aligned}
\sum_{j=0}^{2} \iint_{Q_{T}}\left|\nabla^{j} U_{k}\right|^{2} \leq & C \iint_{Q_{T}}\left|h_{k}\right|^{2} \\
& +C \sum\left\|Z_{k}(\cdot, 0)\right\|_{W^{2,2}(\Omega)} \leq C \quad \text { (by (4.5), (1.14)). }
\end{aligned}
$$

Consequently, by Sobolev's imbedding,

$$
\iint_{Q_{r}}\left|\nabla U_{k}\right|^{r} \leq C \quad \forall r<\tilde{p}
$$

Next, by Lemma 2.1 and (4.5),

$$
\iint_{Q_{T}}\left|\nabla V_{k}\right|^{r} \leq \sum_{j} \iint_{Q_{T}}\left|g_{j k, 1}\right|^{r} \leq C \quad \forall r<\tilde{p} .
$$

Consider the functions $\tilde{Z}_{k}=Z_{k}-U_{k}-V_{k}$. From (4.2), (4.3) and (4.4)-(4.6) we see that the $\tilde{Z}_{k}$ satisfy:

$$
\begin{aligned}
\frac{\partial}{\partial t} \tilde{Z}_{k}-\gamma \Delta \tilde{Z}_{k}= & -\sum \frac{\partial}{\partial x_{i}}\left(\left(\gamma \delta_{i j} \delta_{k l}-A_{k l}^{i j}(q)\right) \frac{\partial \tilde{Z}_{l}}{\partial x_{j}}\right) \\
& +\sum \frac{\partial}{\partial x_{j}} \hat{g}_{k j} \text { in } Q_{T}
\end{aligned}
$$

$$
\tilde{Z}_{k}=0 \quad \text { on } \partial_{p} Q_{T}
$$

where

$$
\begin{aligned}
\hat{g}_{k j}= & -\sum\left(\gamma \delta_{i j} \delta_{k l}-A_{k l}^{i j}(q) \frac{\partial\left(U_{l}+V_{l}\right)}{\partial x_{j}}\right. \\
& +\sum B_{k l}^{i j}(p) \frac{\partial}{\partial x_{j}}\left(\eta \frac{\partial u_{l}}{\partial x_{m}}\right)
\end{aligned}
$$


In order to estimate $\nabla \tilde{Z}_{k}$ we consider, more generally, the system

$$
\begin{gathered}
\dot{Y}_{k}-\gamma \Delta Y_{k}=\sum_{i, j, l} \frac{\partial}{\partial x_{i}}\left(\left(\gamma \delta_{i j} \delta_{k l}-A_{k l}^{i j}(q)\right) h_{l j}\right) \\
+\sum \frac{\partial}{\partial x_{j}} g_{k j} \text { in } Q_{T} \\
Y_{k}=0 \text { on } \partial_{p} Q_{T}
\end{gathered}
$$

and proceed as in $\S 2$ to define an operator $S$ mapping vectors $G=$ $\left(h_{l j}, g_{k j}\right)$ into vectors $\nabla Y=\left(\nabla Y_{1}, \ldots, \nabla Y_{N}\right)$ where $Y_{1}, \ldots, Y_{N}$ is the solution of (4.13), (4.14); we use the $L^{s}$ norms

$$
\begin{gathered}
\|G\|=\left\{\iint_{Q_{T}}\left(\sum\left|h_{l j}\right|^{s}+M \sum\left|g_{k j}\right|^{s}\right)\right\}^{1 / s}, \\
\|\nabla Y\|_{s}=\left\{\iint_{Q_{T}} \sum\left|\nabla Y_{k}\right|^{s}\right\}^{1 / s} .
\end{gathered}
$$

By Lemma 2.1

$$
\|S\|_{s} \leq A_{s},
$$

where $A_{s}$ is a constant. Using (2.10) we can derive the same estimate (2.16) as before, provided $M$ is sufficiently large, and then (2.18) also holds. Recalling (4.10)-(4.12) we deduce that if $p_{0}>2, p_{0}-2$ small enough, then

$$
\sum \iint_{Q_{T}}\left|\nabla \tilde{Z}_{k}\right|^{s} \leq C \sum \iint_{Q_{T}}\left|\hat{g}_{k j}\right|^{s} \quad \forall 2 \leq s \leq p_{0}
$$

This inequality can also be established with $Q_{T}$ replaced by $Q_{t}$, for any $0<t \leq T$. Substituting $\hat{g}_{k i}$ from (4.12) and using (4.8) (4.9), we get

$$
\sum \iint_{Q_{T}}\left|\nabla Z_{k}\right|^{s} \leq C+\sum \iint_{Q_{t}}\left|\nabla\left(\eta \frac{\partial u_{k}}{\partial x_{m}}\right)\right|^{s}, p_{2 \leq 2 \leq p_{0}}
$$

Hence, by Gronwall's inequality (cf. the argument following (2.19)),

$$
\sum \iint_{Q_{r}}\left|\nabla Z_{k}\right|^{s} \leq C
$$

It follows that

$$
\sum_{k} \iint_{Q_{T}}\left|\nabla\left(\eta \frac{\partial \dot{u}_{k}}{\partial x_{m}}\right)\right|^{s}+\sum_{k} \iint_{Q_{T}}\left|\nabla\left(\eta \frac{\partial u_{k}}{\partial x_{m}}\right)\right|^{s} \leq C .
$$


In deriving (4.17) we have assumed that $\nabla^{2} \dot{u}_{k}, \nabla^{2} u_{k}$ belong to $L^{s}$. In order to derive (4.17) without making this a priori restriction, we work with finite differences, as in $\S 3$, and establish (4.17) with $\partial / \partial x_{m}$ replaced by $\Delta_{m}^{h}$. It then follows that $\nabla^{2} \dot{u}_{k}, \nabla^{2} u_{k}$ belong to $L^{s}\left(\Omega_{0} \times(0, T)\right)$ for any compact subdomain $\Omega_{0}$ of $\Omega$, and (4.17) holds. In order to derive the $L^{s}$ estimates near the lateral boundary of $Q_{T}$, we apply the same arguments used in the derivation of (4.17) to the system (3.3), and thus derive $L^{s}$ estimates, which extend the $L^{2}$ estimates of (3.14). Finally, using (3.17), we can estimate the $L^{s}$ norm of $\partial^{2} \dot{U}_{k} / \partial\left(x_{n}^{\prime}\right)^{2}, \partial^{2} U_{k} / \partial\left(x_{n}^{\prime}\right)^{2}$ near the boundary. This completes the proof of Theorem 4.1.

Analogously to Corollary 2.3 we have:

COROLlary 4.2. For any $2<p_{0}<q_{0}$, if $\lambda_{2}-\lambda_{1}$ is sufficiently small so that $\vartheta_{p_{0}}<1\left(\vartheta_{p_{0}}\right.$ as in (2.18)) then (4.1) holds for any $2 \leq s \leq p_{0}$.

\section{Additional regularity.}

LEMMA 5.1. If $p_{0}>2$ and $p_{0}-2$ is sufficiently small, then

$$
\sup _{0<t<T} \sum_{k} \int_{\Omega_{t}}\left|\ddot{u}_{k}\right|^{s} d s \leq C \quad \forall 2<s \leq p_{0} .
$$

Proof. Multiplying (2.4) by $\left|\dot{z}_{k}\right|^{\alpha} z_{k}(\alpha=s-2>0)$ and integrating over $Q_{t}$, we obtain

$$
\begin{aligned}
\frac{1}{s} \int_{\Omega_{t}}\left|\ddot{u}_{k}\right|^{s}-\frac{1}{s} \int_{\Omega_{0}}\left|\ddot{u}_{k}\right|^{s} \\
=-(1+\alpha) \iint_{Q_{t}} \sum A_{k l}^{i j}(q) \frac{\partial \ddot{u}_{l}}{\partial x_{j}} \frac{\partial \ddot{u}_{k}}{\partial x_{i}}\left|\ddot{u}_{k}\right|^{\alpha} \\
\quad-(1+\alpha) \iint_{Q_{t}} \sum B_{k l}^{i j}(p) \frac{\partial \dot{u}_{l}}{\partial x_{j}} \frac{\partial \ddot{u}_{k}}{\partial x_{i}}\left|\ddot{u}_{k}\right|^{\alpha}+\iint_{Q_{t}} \dot{f}_{k}\left|\ddot{u}_{k}\right|^{\alpha} \ddot{u}_{k} .
\end{aligned}
$$

By Theorems 2.2, 4.1,

$$
|\nabla \dot{u}||\nabla \ddot{u}| \text { and }|\nabla u||\nabla \ddot{u}|
$$

belong to $L^{r}\left(Q_{t}\right)$ for some $r>1$. Choosing $s=2 r$ and using Hölder's inequality, we get

$$
\sum_{k} \int_{\Omega_{t}}\left|\ddot{u}_{k}\right|^{s} \leq C+C \sum_{k} \iint_{Q_{t}}\left|\ddot{u}_{k}\right|^{s}
$$

and (5.1) then follows by Gronwall's inequality. 
TheOREM 5.2. There exists a $p_{0}>2$ such that

$$
\sup _{0<t<T} \sum_{k} \int_{\Omega}\left|\nabla^{2} \dot{u}_{k}\right|^{s} \leq C \quad \forall 2 \leq s \leq p_{0} .
$$

Proof. Notice that

$$
\left.\sum \int_{\Omega_{t}}\left|\nabla^{2} u_{k}\right|^{s} \leq C+C \sum \iint_{Q_{t}}\left|\nabla^{2} \dot{u}_{k}\right|^{s} \leq C^{\prime} \quad \text { (C' constant }\right)
$$

We can write (1.4) as an elliptic system

$$
-\sum \frac{\partial}{\partial x_{i}} \frac{\partial V(q)}{\partial q_{k i}}=\tilde{g}_{k}
$$

where, by (5.3) and Lemma 5.1,

$$
\sum \int_{\Omega_{t}}\left|\tilde{g}_{k}\right|^{s} \leq C
$$

if $2 \leq s \leq p_{0}$ for some $p_{0}>2$. We now argue as in $\S 4$; we set $z_{k}=\partial \dot{u}_{k} / \partial x_{m}, Z_{k}=\eta z_{k}$ ( $\eta$ a cut-off function for $\left.B_{R^{\prime}}\right)$, differentiate (5.4) with respect to $x_{m}$, multiply by $\eta$ and integrate over $\Omega_{t}$. We obtain, analogously to (4.2),

$$
-\gamma \Delta Z_{k}=-\sum\left(\frac{\partial}{\partial x_{i}}\left(\gamma \delta_{i j} \delta_{k l}-A_{k l}^{i j}(q) \frac{\partial Z_{l}}{\partial x_{j}}\right)+\tilde{G}_{k}\right.
$$

where the $L^{q}\left(\Omega_{t}\right)$-norm of $\tilde{G}_{k}$ can be estimated using (5.5). We then deduce, as in the parabolic case, that (2.18) holds and, consequently,

$$
\int_{\Omega_{t}}\left|\nabla Z_{k}\right|^{s} \leq C \quad \forall 2 \leq s \leq p_{0}
$$

Thus

$$
\sup _{0<t<T} \sum \int_{B_{R}}\left|\nabla^{2} \dot{u}_{k}(x, t)\right|^{s} \leq C \quad \text { if } \bar{B}_{R} \subset \Omega .
$$

Similarly we obtain the corresponding estimate with $B_{R}$ replaced by $B_{R}\left(x_{0}\right) \cap \Omega$, where $x_{0} \in \partial \Omega$, and (5.2) follows.

THEOREM 5.3. If $n \leq 2$ then $\nabla u_{k}$ and $\nabla \dot{u}_{k}$ are Hölder continuous in $\bar{\Omega}_{T} ;$ consequently $\left(u_{1}, \ldots, u_{N}\right)$ is a classical solution in $\bar{\Omega}_{T}$ for all $T>0$.

Proof. From Theorem 5.2 and Sobolev's imbedding it follows that

$$
\left|\nabla \dot{u}\left(x_{1}, t\right)-\nabla \dot{u}\left(x_{2}, t\right)\right| \leq C\left|x_{1}-x_{2}\right|^{2} \quad(\alpha=1-2 / s) .
$$


We next prove that, for any $0<t_{1}<t_{2}<T$,

$$
\left|\nabla \dot{u}\left(x, t_{2}\right)-\nabla \dot{u}\left(x, t_{1}\right)\right| \leq C\left|t_{2}-t_{1}\right|^{\beta} \quad(\beta=(1-2 / s)(1-1 / s)) .
$$

Let $\rho=\left(t_{2}-t_{1}\right)^{\gamma}(\gamma=1-1 / s)$ and consider first the case where $B_{\rho}(x)$ is contained in $\Omega$. Let

$$
v(x, t)=\frac{\partial^{2} u_{k}}{\partial t \partial x_{m}}, \quad v_{\rho}(x, t)=f_{B_{\rho}(x)} v(y, t) d y
$$

where $f$ means the average. Then

$$
\begin{gathered}
\left|v\left(x, t_{2}\right)-v\left(x, t_{1}\right)\right| \leq \sum_{i=1}^{2}\left|v\left(x, t_{i}\right)-v_{\rho}\left(x, t_{i}\right)\right|+\left|v_{\rho}\left(x, t_{2}\right)-v_{\rho}\left(x, t_{1}\right)\right| \\
\leq C \rho^{\alpha}+\left|B_{\rho}\right|^{-1} \int_{t_{1}}^{t_{2}} \int_{B_{\rho}(x)}\left|v_{t}(y, t)\right| \quad(\text { by }(5.6)) \\
\leq C \rho^{\alpha}+\left|t_{2}-t_{1}\right|^{1-1 / s}\left|B_{\rho}\right|^{-1 / s}\left(\iint_{Q_{T}}\left|v_{t}\right|^{s}\right)^{1 / s} \\
\quad+C\left|t_{2}-t_{1}\right|^{\gamma(1-2 / s)}+C\left|t_{2}-t_{1}\right|^{1-1 / s-2 \gamma / s}
\end{gathered}
$$

by Theorem 2.2 , and (5.7) follows. In case $B_{\rho}(x)$ is not contained in $\Omega$, we work with

$$
f_{B_{\rho}(x) \cap \Omega} v(y, t) d y \text { instead of } f_{B_{\rho}(x)} v(y, t) d y .
$$

The Hölder continuity of $\nabla \dot{u}$ follows from (5.6), (5.7). The Hölder continuity of $\nabla u$ follows from that of $\nabla \dot{u}$, and thus the proof of Theorem 5.3 is complete.

Using Corollaries 2.3, 4.2 and an extension of Theorem 5.2 to any $p_{0}\left(p_{0}>n\right)$ if $\lambda_{2}-\lambda_{1}$ is small enough we also have:

THEOREM 5.4. Let $n \geq 3$. If $\lambda_{2}-\lambda_{1}$ is small enough then $\nabla u_{k}$ and $\nabla \dot{u}_{k}$ are Hölder continuous in $\bar{Q}_{T}$ and, consequently, $\left(u_{1}, \ldots, u_{N}\right)$ is a classical solution in $\bar{\Omega}_{T}$.

6. General $W$ and linear viscosity term. In this section we consider systems of the form

$$
\ddot{u}_{k}-\sum_{i=1}^{n} \frac{\partial}{\partial x_{i}} \frac{\partial W(p)}{\partial p_{k i}}-\Delta \dot{u}=f_{k} \quad(1 \leq k \leq N)
$$


instead of (1.4), but replace the condition (1.2) by a much weaker condition which allows quite general nonlinear growth for $W$, namely:

$$
\begin{aligned}
\tilde{\lambda}_{1}(\delta+|p|)^{r-2}|\xi|^{2} \leq & \sum_{k, l=1}^{N} \sum_{i, j=1}^{n} \frac{\partial^{2} W(p)}{\partial p_{k i} \partial p_{l j}} \xi_{i}^{k} \xi_{j}^{l} \\
\leq & \tilde{\lambda}_{2}(\delta+|p|)^{r-2}|\xi|^{2}, \\
& \quad \text { where } 0<\tilde{\lambda}_{1}<\tilde{\lambda}_{2}<\infty, \delta \geq 0, r>2 .
\end{aligned}
$$

We also suppose that

$$
W(0) \geq 0, \quad \frac{\partial W(0)}{\partial p_{k i}}=0
$$

then $W(p)>0$ if $|p|>0$.

DEFINITION 6.1. A strong solution $u=\left(u_{1}, \ldots, u_{N}\right)$ of (6.1), (1.5), (1.6) in $Q_{T}$ is a function $u$ satisfying: (i) the first two relations in (1.8) hold; (ii) $W(\nabla u) \in L^{\infty}\left((0, T) ; L^{1}(\Omega)\right)$; (iii) (6.1) holds in the distribution since in $Q_{T}$ and (1.6) holds in the trace sense; finally, (iv) the functions

$$
\ddot{u}_{k}, \quad \nabla^{2} \dot{u}_{k}, \quad \frac{\partial}{\partial x_{i}} \frac{\partial W(p)}{\partial p_{k i}}
$$

belong to $L_{\text {loc }}^{r^{\prime}}\left(Q_{T}\right)$ and (6.1) holds pointwise a.e.; here $1 / r^{\prime}+1 / r=1$.

TheOREM 6.1. Assume that (1.12)-(1.14) and (6.2), (6.3) hold. Then there exists a function $u=\left(u_{1}, \ldots, u_{N}\right)$ which is a strong solution of (6.1), (1.5), (1.6) in $Q_{T}$, for every $T>0$.

Proof. We begin by introducing a family of truncations of $W$. For any small $\varepsilon>0$, let $M=[1 / \varepsilon]$ (the largest integer $\leq 1 / \varepsilon$ ), and define

$$
W_{\varepsilon}(p)=W(p) \eta_{\varepsilon}(|p|)+C_{0} M^{r-2}|p|^{2} \chi_{\varepsilon}(|p|)+\varepsilon|p|^{2}
$$

where $\eta_{\varepsilon}(M s)=\phi(s), \chi_{\varepsilon}(M s)=\psi(s), \phi \in C^{3}\left(\mathbf{R}^{1}\right), \phi(s)=1$ if $s \leq \beta$, $\phi(s)=0$ if $s \geq \gamma$ and $\psi \in C^{3}(\mathbf{R}), \psi(s)=0$ if $s \leq \alpha, \psi(s)=1$ if $s \geq \beta$, $\psi^{\prime}(s) \geq 0$ if $\alpha<s<\beta$, and

$$
2 \psi(s)+s \psi^{\prime}(s)+s^{2} \psi^{\prime \prime}(s) \geq 0 \text { if } \alpha<s<\beta .
$$

We can choose, for instance

$$
\alpha=e^{\pi /(4 \sqrt{2})}, \quad \beta=e^{\pi / \sqrt{2}}, \quad \gamma=\beta+1
$$

and take $\psi_{0}(s)=(\mu-\cos (\sqrt{2} \log s))^{+} /(1+\mu)$ if $\alpha \leq s \leq \beta$, where $\mu$ is a sufficiently small positive constant, and then mollify $\psi_{0}$ and shift 
it slightly to the left to obtain a function $\psi$ satisfying all of the above conditions.

Using (6.5) we compute that

$$
\sum \frac{\partial^{2}\left(\chi_{\varepsilon}(|p|)|p|^{2}\right)}{\partial p_{k i} \partial p_{l j}} \xi_{i}^{k} \xi_{j}^{l} \geq 0 \text { if }|p| \leq \beta M
$$

and, therefore, if $C_{0}$ is chosen large enough (independently of $\varepsilon$ ),

$$
\lambda_{1} \Gamma_{\varepsilon}(p)|\xi|^{2} \leq \sum \frac{\partial^{2} W_{\varepsilon}(p)}{\partial p_{k i} \partial p_{l j}} \xi_{i}^{k} \xi_{j}^{l} \leq \lambda_{2} \Gamma_{\varepsilon}(p)|\xi|^{2}
$$

where $\lambda_{1}, \lambda_{2}$ are positive constants independent of $\varepsilon$ and

$$
\Gamma_{\varepsilon}(p)= \begin{cases}(\delta+|p|)^{r-2}+\varepsilon & \text { if }|p|<\gamma M \\ (\delta+\gamma M)^{r-2}+\varepsilon & \text { if }|p| \geq \gamma M\end{cases}
$$

We can also easily verify that

$$
\begin{gathered}
\Gamma_{\varepsilon}(p)^{r^{\prime} /\left(2-r^{\prime}\right)} \leq C\left(1+W_{\varepsilon}(p)\right), \\
\left|\frac{\partial W_{\varepsilon}(p)}{\partial p_{k i}}\right| \leq C\left[W_{\varepsilon}(p) \Gamma_{\varepsilon}(p)\right]^{1 / 2},
\end{gathered}
$$

and, as $\varepsilon \rightarrow 0$,

$$
W_{\varepsilon}(p) \rightarrow W(p) \quad \text { in } C_{\mathrm{loc}}^{3}\left(\mathbf{R}^{n N}\right) .
$$

Consider the system

$$
\ddot{u}_{k}-\sum_{i=1}^{n} \frac{\partial}{\partial x_{i}} \frac{\partial W_{\varepsilon}(p)}{\partial p_{k i}}-\Delta \dot{u}_{k}=f_{k} \quad(1 \leq k \leq N) .
$$

Since $W_{\varepsilon}$ is a $C^{3}$ function, we can apply Theorem 5.4 to conclude that there exists a function $u^{\varepsilon}=\left(u_{1}^{\varepsilon}, \ldots, u_{N}^{\varepsilon}\right)$ which is a classical solution of (6.11), (1.5), (1.6) in $Q_{\infty}$. We shall proceed to derive estimates on $u^{\varepsilon}$ which are independent of $\varepsilon$, and then complete the proof of the theorem by taking $\varepsilon \rightarrow 0$.

First, analogously to the derivation of (1.11) we have

$$
\frac{1}{2} \int_{\Omega_{t}}\left|\dot{u}^{\varepsilon}\right|^{2} d x+\int_{\Omega_{t}} W_{\varepsilon}\left(\nabla u^{\varepsilon}\right) d x+\iint_{Q_{T}}\left|\nabla \dot{u}^{\varepsilon}\right|^{2} d x d t \leq C .
$$

We introduce a function $\eta \in C^{2}(\bar{\Omega})$ satisfying:

$$
c_{1} \operatorname{dist}(x, \partial \Omega) \leq \eta(x) \leq c_{2} \operatorname{dist}(x, \partial \Omega)
$$

where $c_{1}, c_{2}$ are positive constants. 
LEMMA 6.2. For any $T>0$,

$$
\begin{aligned}
\sum_{m} \iint_{Q_{T}} \sum_{i, j, k, l} \frac{\partial^{2} W_{\varepsilon}\left(p^{\varepsilon}\right)}{\partial p_{k i} \partial p_{l j}} & \frac{\partial^{2} u^{\varepsilon}}{\partial x_{i} \partial x_{m}} \frac{\partial^{2} u^{\varepsilon}}{\partial x_{j} \partial x_{m}} \eta^{2} \\
& +\iint_{Q_{T}}\left|\nabla^{2} u^{\varepsilon}\right|^{2} \eta^{2} \leq C
\end{aligned}
$$

where $p^{\varepsilon}=\nabla u^{\varepsilon}$ and $C$ is a constant independent of $\varepsilon$.

Proof. We shall prove that

$$
\begin{aligned}
& \sum_{m} \iint_{Q_{T}}(T-t) \sum_{i, j, k, l} \frac{\partial^{2} W_{\varepsilon}\left(p^{\varepsilon}\right)}{\partial p_{k i} \partial p_{l j}} \frac{\partial^{2} u_{k}^{\varepsilon}}{\partial x_{i} \partial x_{m}} \frac{\partial^{2} u_{l}^{\varepsilon}}{\partial x_{j} \partial x_{m}} \eta^{2} \\
& \quad+\iint_{Q_{T}}\left|\nabla u^{\varepsilon}\right|^{2} \eta^{2} \\
& \quad \leq C\left\{\sum_{0 \leq t \leq T} \int_{\Omega_{t}}\left|\nabla u^{\varepsilon}\right|^{2}+\iint_{Q_{T}}\left|\nabla \dot{u}^{\varepsilon}\right|^{2}+\iint_{Q_{T}} W_{\varepsilon}\left(p^{\varepsilon}\right)+C_{1}\right\}
\end{aligned}
$$

where $C_{1}$ depends only on the initial data and on $f_{k}$. Since the righthand side of (6.15) is bounded for every $T>0$ (by (6.12)) and since (6.15) holds for any $T>0$, the assertion (6.14) then follows.

To prove (6.15) we multiply $(6.11)$ by $\eta^{2}\left(\partial^{2} u_{k}^{\varepsilon} / \partial x_{m}^{2}\right)$ and integrate over $Q_{t}$, then sum over $k, m$, and integrate once more in $t$. We compute the separate terms, dropping usually the index $\varepsilon$. First,

$$
\begin{aligned}
\sum_{m, k} \iint_{Q_{t}} \ddot{u}_{k} \frac{\partial^{2} u_{k}}{\partial x_{m}^{2}} \eta^{2}= & -\int_{\Omega_{t}} \sum \frac{\partial \dot{u}_{k}}{\partial x_{m}} \eta^{2}+\int_{\Omega_{0}} \sum \frac{\partial \dot{u}_{k}}{\partial x_{m}} \frac{\partial u_{k}}{\partial x_{m}} \eta^{2} \\
& +\iint_{Q_{t}} \sum \frac{\partial \dot{u}_{k}}{\partial x_{m}} \frac{\partial u_{k}}{\partial x_{m}} \eta^{2}-2 \int_{\Omega_{t}} \sum \dot{u}_{k} \frac{\partial u_{k}}{\partial x_{m}} \eta \eta_{x_{m}} \\
& +2 \int_{\Omega_{0}} \sum \dot{u}_{k} \frac{\partial u_{k}}{\partial x_{m}} \eta \eta_{x_{m}}+2 \iint_{Q_{t}} \dot{u}_{k} \frac{\partial \dot{u}_{k}}{\partial x_{m}} \eta \eta_{x_{m}} .
\end{aligned}
$$

Integrating over $(0, T)$ we find that

$$
\int_{0}^{T} d t \sum_{m, k} \iint_{Q_{1}} \ddot{u}_{k} \frac{\partial^{2} u_{k}}{\partial x_{m}^{2}} \eta^{2} \text { is bounded by the }
$$

right-hand side of (6.15). 
Next,

$$
\begin{aligned}
-\sum & \iint_{Q_{t}} \sum \frac{\partial}{\partial x_{i}}\left(\frac{\partial W}{\partial p_{k i}}\right) \frac{\partial^{2} u_{k}}{\partial x_{m}^{2}} \eta^{2} \\
= & -\iint_{Q_{i}} \sum \frac{\partial^{2} W}{\partial p_{k i} \partial p_{l j}} \frac{\partial^{2} u_{k}}{\partial x_{i} \partial x_{m}} \frac{\partial^{2} u_{l}}{\partial x_{j} \partial x_{m}} \eta^{2} \\
& -\iint_{Q_{t}} \sum \frac{\partial W}{\partial p_{k i}} \frac{\partial^{2} u_{k}}{\partial x_{i} \partial x_{m}} \eta \eta_{x_{m}}+2 \iint_{Q_{i}} \sum \frac{\partial W}{\partial p_{k i}} \frac{\partial^{2} u_{k}}{\partial x_{m}^{2}} \eta \eta_{x_{i}} .
\end{aligned}
$$

In view of (6.8), (6.9), each of the last two integrals is bounded by

$$
\begin{aligned}
& C\left\{\iint_{Q_{t}} \Gamma_{\varepsilon}(p)\left|\nabla^{2} u\right|^{2} \eta^{2}\right\}^{1 / 2}\left\{\iint_{Q_{t}} W_{\varepsilon}\right\}^{1 / 2} \\
& \quad \leq \mu \iint_{Q_{t}} \sum \frac{\partial^{2} W}{\partial p_{k i} \partial p_{l j}} \frac{\partial^{2} u_{k}}{\partial x_{i} \partial x_{m}} \frac{\partial^{2} u_{l}}{\partial x_{j} \partial x_{m}} \eta^{2}+C_{\mu} \iint_{Q_{t}} W
\end{aligned}
$$

for any small positive constant $\mu$. Hence

$$
\begin{array}{rl}
(6.17)-\int_{0}^{T} & d t \sum_{m, k} \iint_{Q_{i}} \sum \frac{\partial}{\partial x_{i}}\left(\frac{\partial W}{\partial p_{k i}}\right) \frac{\partial^{2} u_{k}}{\partial x_{m}^{2}} \eta^{2} \\
= & -(1-\tilde{\mu}) \iint_{Q_{T}}(T-t) \sum \frac{\partial^{2} W}{\partial p_{k i} \partial p_{l j}} \frac{\partial^{2} u_{k}}{\partial x_{i} d x_{m}} \frac{\partial^{2} u_{l}}{\partial x_{j} \partial x_{m}} \\
& +\tilde{C} \iint_{Q_{T}} W
\end{array}
$$

where $|\tilde{\mu}| \leq 1 / 2$ and $\tilde{C}$ is a constant bounded independently of $\varepsilon$.

Finally,

$$
\begin{aligned}
-\sum & \iint_{Q_{i}} \Delta \dot{u}_{k} \frac{\partial^{2} u_{k}}{\partial x_{m}^{2}} \eta^{2} \\
= & \iint_{Q_{t}} \sum \nabla \dot{u}_{k} \nabla\left(\frac{\partial^{2} u_{k}}{\partial x_{m}^{2}}\right) \eta^{2}+2 \iint_{Q_{t}} \sum \nabla \dot{u}_{k} \frac{\partial^{2} u_{k}}{\partial x_{m}^{2}} \eta \nabla \eta \\
= & -\frac{1}{2} \int_{\Omega_{t}} \sum\left|\nabla \frac{\partial u_{k}}{\partial x_{m}}\right|^{2} \eta^{2}+\frac{1}{2} \int_{\Omega_{0}} \sum\left|\nabla \frac{\partial u_{k}}{\partial x_{m}}\right|^{2} \eta^{2} \\
& -2 \iint_{Q_{t}} \sum \nabla \dot{u}_{k} \nabla\left(\frac{\partial u_{k}}{\partial x_{m}}\right) \eta \eta_{x_{m}}+2 \iint_{Q_{t}} \sum \nabla \dot{u}_{k} \frac{\partial^{2} u_{k}}{\partial x_{m}^{2}} \eta \nabla \eta .
\end{aligned}
$$

Integrating over $(0, T)$ we obtain

(6.18) $-\int_{0}^{T} d t \sum_{m, k} \iint_{Q_{t}} \Delta \dot{u}_{k} \frac{\partial^{2} u_{k}}{\partial x_{m}^{2}} \eta^{2}=-\frac{1}{2} \iint_{Q_{r}}\left|\nabla^{2} u\right|^{2} \eta^{2}+I_{\varepsilon}$

where $I_{\varepsilon}$ is bounded by the right-hand side of (6.15). 
Combining (6.18) with (6.17), (6.16), the estimate (6.15) follows.

Corollary 6.3. For any $h, k, l, i, j, m, s$

$$
\left\|\frac{\partial^{2} W_{\varepsilon}}{\partial p_{k i} \partial p_{l j}} \frac{\partial^{2} u_{h}^{\varepsilon}}{\partial x_{m} \partial x_{s}} \eta\right\|_{L^{r J}\left(Q_{r}\right)} \leq C .
$$

Indeed, by (6.6), (6.8) and Lemma 6.2, the left-hand side of (6.19) is bounded by

$$
c\left\{\iint_{Q_{T}} \Gamma_{\varepsilon}\left(p_{\varepsilon}\right)\left|\nabla^{2} u^{\varepsilon}\right|^{2} \eta^{2}\right\}^{r^{\prime}}\left\{1+\iint_{Q_{T}} W_{\varepsilon}\left(p_{\varepsilon}\right)\right\}^{1-r^{\prime}} \leq C .
$$

Consider the functions $v_{k}^{\varepsilon}=u_{k}^{\varepsilon} \eta$. Clearly

$$
\ddot{v}_{k}^{\varepsilon}-\Delta \dot{v}_{k}^{\varepsilon}=f_{k} \eta-\sum \frac{\partial^{2} W_{\varepsilon}\left(p_{\varepsilon}\right)}{\partial p_{k i} \partial p_{l j}} \frac{\partial^{2} u_{l}^{\varepsilon}}{\partial x_{i} \partial x_{j}} \eta+g_{k} \equiv G_{k}
$$

where

$$
g_{k}=-(\Delta \eta) \dot{u}_{k}-2 \nabla \eta \nabla \dot{u}_{i}
$$

By (6.12) and Corollary 6.3,

$$
\left\|G_{k}\right\|_{L^{r^{\prime}}\left(Q_{T}\right)} \leq C,
$$

and, by $L^{r^{\prime}}$ parabolic estimates [9],

$$
\begin{aligned}
& \iint_{Q_{T}}\left|\ddot{v}_{k}^{\varepsilon}\right|^{r^{\prime}}+\iint_{Q_{T}}\left|\nabla^{2} \dot{v}_{k}^{\varepsilon}\right|^{r^{\prime}} \\
& \leq C\left(\iint_{Q_{T}}\left|G_{k}\right|^{r^{\prime}}+\int_{\Omega_{0}}\left|\nabla^{2} \dot{v}_{k}^{\varepsilon}\right|^{r^{\prime}}\right) \leq C .
\end{aligned}
$$

Using the estimates (6.12), (6.14), (6.23), we can extract a sequence $\varepsilon=\varepsilon_{m} \rightarrow 0$ such that $\ddot{v}^{\varepsilon} \rightarrow \ddot{v}, \nabla^{2} \dot{v}^{\varepsilon} \rightarrow \nabla^{2} \dot{v}$ weakly in $L^{r^{\prime}}\left(Q_{T}\right)$ for any $T>0$ and, further, since

$$
\left\|\nabla v^{\varepsilon}\right\|_{W^{1, r^{\prime}}\left(Q_{r}\right)} \leq C,
$$

we may suppose that $\nabla v^{\varepsilon} \rightarrow \nabla v$ in $L^{r^{\prime}}\left(Q_{T}\right)$ and a.e. But then we have

$$
\frac{\partial W_{\varepsilon}\left(p_{\varepsilon}\right)}{\partial p_{k i}} \rightarrow \frac{\partial W(p)}{\partial p_{k i}} \text { a.e. }
$$

and strongly in $L^{s}\left(Q_{T}\right)$ for some $s>1$ (by (6.8), (6.9) and (6.12)). Hence, by Corollary 6.3,

$$
\frac{\partial}{\partial x_{i}} \frac{\partial W(p)}{\partial p_{k i}} \in L_{\mathrm{loc}}^{r^{\prime}}\left(Q_{T}\right)
$$


Since, by (6.24), $p \equiv \nabla u \in W_{\text {loc }}^{l, r^{\prime}}\left(Q_{T}\right)$, the distribution derivative in (6.25) can be identified with the function

$$
\sum \frac{\partial^{2} W(p)}{\partial p_{k i} \partial p_{l j}} \frac{\partial^{2} u_{l}}{\partial x_{i} \partial x_{j}}
$$

One can now easily check that $u$ satisfies all the properties of a strong solution.

REMARK 6.1. All the results of $\S \S 1-5$ extend to the case where $W=W(x, u, p), V=V(x, u, q)$; the results of $\S 6$ extend to the case where $\Delta \dot{u}$ is replaced by any linear elliptic operator $L \dot{u}$ with smooth coefficients.

\section{REFERENCES}

[1] G. Andrew, On the existence of solutions to the equation $u_{t t}=u_{x x t}+\sigma\left(u_{x}\right)_{x}, \mathrm{~J}$. Differential Equations, 35 (1980), 200-231.

[2] C. M. Dafermos, The mixed initial-boundary value problem for the equations of nonlinear one-dimensional visco-elasticity, J. Differential Equations, 6 (1969), 71-86.

[3] H. Engler, Strong solutions for strongly damped quasilinear wave equations, Contemporary Math., 64 (1987), 219-237.

[4] W. N. Findleky, J. S. Lai and K. Onaran, Creep and Relaxation of Nonlinear Viscoelastic Materials, North Holland, Amsterdam, New York, 1976.

[5] H. Gajewski, K. Groger and K. Zacharias, Nonlinear Operator Equations and Operator Differential Equations, Akademy Verlag, Berlin, 1974.

[6] J. M. Greenberg, R. C. MacCamy and V. J. Mizel, On the existence, uniqueness and stability of the equation $\sigma^{\prime}\left(u_{x}\right) u_{x x}+\lambda u_{x x t}=\rho_{0} u_{t t}$, J. Math. Mech., 17 (1968), 707-728.

[7] J. M. Greenberg, On the existence, uniqueness and stability of the equation $\rho_{0} X_{t t}=E\left(X_{x}\right)+\lambda X_{x x t}$, J. Math. Anal. Appl., 25 (1969), 575-591.

[8] J. K. Knowles, One finite antiplane shear for incomprehensible elastic materials, J. Austral. Math. Soc. Ser. B, 19 (1975/76), 400-415.

[9] O. A. Ladyzenskaja, V. A. Solonnikov and N. N. Uralceva, Linear and Quasilinear Equations of Parabolic Type, Transl. Amer. Math. Soc., Providence, R.I., 1968.

[10] H. Petcher, On global regular solution of third partial differential equations, J. Math. Anal. Appl., 73 (1980), 278-299.

[11] M. Potier-Ferry, On the mathematical foundation of elastic stability, I. Archive Rat. Mech. Anal., 78 (1982), 55-72.

Received April 24, 1987. This work is partially supported by National Science Foundation Grants DMS-8420896 and DMS-8501397.

Purdue University

WEST LAFAyETTE, IN 47907 



\section{PACIFIC JOURNAL OF MATHEMATICS}

\section{EDITORS}

\author{
V. S. VARADARAJAN \\ (Managing Editor) \\ University of California \\ Los Angeles, CA 90024 \\ HeRBERT ClEMENS \\ University of Utah \\ Salt Lake City, UT 84112 \\ R. FINN \\ Stanford University \\ Stanford, CA 94305
}

\section{HERMANN FLASCHKA \\ University of Arizona \\ Tucson, AZ 85721}

VAUghan F. R. Jones

University of California

Berkeley, CA 94720

ROBION KIRBY

University of California

Berkeley, CA 94720

\author{
C. C. MOORE \\ University of California \\ Berkeley, CA 94720
}

HAROLD STARK

University of California, San Diego

La Jolla, CA 92093

\section{ASSOCIATE EDITORS}
R. ARENS
E. F. BECKENBACH
B. H. NEUMANN
F. WOLF
K. YOSHIDA
(1906-1982)

\section{SUPPORTING INSTITUTIONS}

\begin{abstract}
UNIVERSITY OF ARIZONA
UNIVERSITY OF BRITISH COLUMBIA

UNIVERSITY OF CALIFORNIA

MONTANA STATE UNIVERSITY

UNIVERSITY OF NEVADA, RENO

NEW MEXICO STATE UNIVERSITY

OREGON STATE UNIVERSITY
\end{abstract}
CALIFORNIA INSTITUTE OF TECHNOLOGY

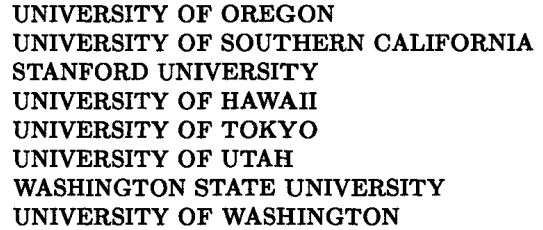

The Supporting Institutions listed above contribute to the cost of publication of this Journal, but they are not owners or publishers and have no responsibility for its content or policies.

Mathematical papers intended for publication in the Pacific Journal of Mathematics should be in typed form or offset-reproduced (not dittoed), double spaced with large margins. Please do not use built up fractions in the text of the manuscript. However, you may use them in the displayed equations. Underline Greek letters in red, German in green, and script in blue. The first paragraph must be capable of being used separately as a synopsis of the entire paper. In particular it should contain no bibliographic references. Please propose a heading for the odd numbered pages of less than 35 characters. Manuscripts, in triplicate, may be sent to any one of the editors. Please classify according to the scheme of Math. Reviews, Index to Vol. 39. Supply name and address of author to whom proofs should be sent. All other communications should be addressed to the managing editor, or Elaine Barth, University of California, Los Angeles, California 90024.

There are page-charges associated with articles appearing in the Pacific Journal of Mathematics. These charges are expected to be paid by the author's University, Government Agency or Company. If the author or authors do not have access to such Institutional support these charges are waived. Single authors will receive $\mathbf{5 0}$ free reprints; joint authors will receive a total of 100 free reprints. Additional copies may be obtained at cost in multiples of 50 .

The Pacific Journal of Mathematics is issued monthly as of January 1966. Regular subscription rate: $\$ 190.00$ a year (5 Vols., 10 issues). Special rate: $\$ 95.00$ a year to individual members of supporting institutions.

Subscriptions, orders for numbers issued in the last three calendar years, and changes of address should be sent to Pacific Journal of Mathematics, P.O. Box 969, Carmel Valley, CA 93924, U.S.A. Old back numbers obtainable from Kraus Periodicals Co., Route 100, Millwood, NY 10546.

The Pacific Journal of Mathematics at P.O. Box 969, Carmel Valley, CA 93924 (ISSN 0030-8730) publishes 5 volumes per year. Application to mail at Second-class postage rates is pending at Carmel Valley, California, and additional mailing offices. Postmaster: send address changes to Pacific Journal of Mathematics, P.O. Box 969, Carmel Valley, CA 93924.

PUBLISHED BY PACIFIC JOURNAL OF MATHEMATICS, A NON-PROFIT CORPORATION Copyright (C) 1988 by Pacific Journal of Mathematics 


\section{Pacific Journal of Mathematics}

Vol. 135, No. $1 \quad$ September, 1988

Margaret M. Bayer, Barycentric subdivisions $\ldots \ldots \ldots \ldots \ldots \ldots \ldots \ldots \ldots$

Eung Chun Cho, $s$-Smith equivalent representations of dihedral groups . . . . 17

Avner Friedman and Jindrich Necas, Systems of nonlinear wave equations

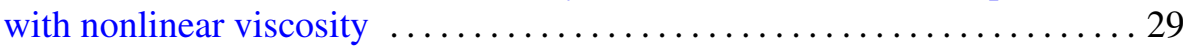

Kyong Taik Hahn, Nontangential limit theorems for normal mappings . . . . 557

Eloise A. Hamann, Evan Green Houston, Jr. and Jon Lee Johnson,

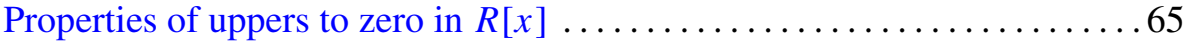

José M. Souto Menéndez, On a theorem due to Cassels ............. 81

Courtney Hughes Moen, Irreducibility of unitary principal series for

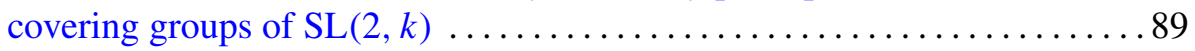

Frank M. Neubrander, Integrated semigroups and their applications to the

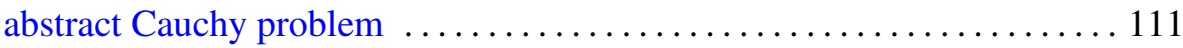

C. K. Qu and Roderick Sue-Chuen Wong, Szegő's conjecture on Lebesgue

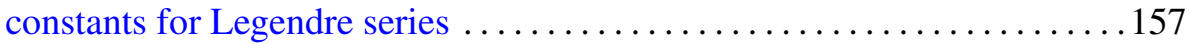

John Brendan Sullivan, The Euler character and cancellation theorems for

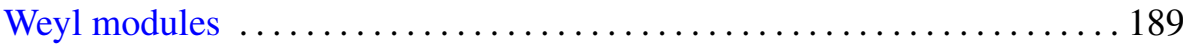

